\title{
Altered chromatin compaction and histone methylation drive non-additive gene expression in an interspecific Arabidopsis hybrid
}

Wangsheng Zhu' ${ }^{1 \dagger}$, Bo Hu², Claude Becker ${ }^{1,3}$, Ezgi Süheyla Doğan², Kenneth Wayne Berendzen², Detlef Weigel ${ }^{1 *}$ and Chang Liu ${ }^{1,2^{*+}}$ (D)

\begin{abstract}
Background: The merging of two diverged genomes can result in hybrid offspring that phenotypically differ greatly from both parents. In plants, interspecific hybridization plays important roles in evolution and speciation. In addition, many agricultural and horticultural species are derived from interspecific hybridization. However, the detailed mechanisms responsible for non-additive phenotypic novelty in hybrids remain elusive.

Results: In an interspecific hybrid between Arabidopsis thaliana and A. lyrata, the vast majority of genes that become upregulated or downregulated relative to the parents originate from A. thaliana. Among all differentially expressed $A$. thaliana genes, the majority is downregulated in the hybrid. To understand why parental origin affects gene expression in this system, we compare chromatin packing patterns and epigenomic landscapes in the hybrid and parents. We find that the chromatin of A. thaliana, but not that of A. lyrata, becomes more compact in the hybrid. Parental patterns of DNA methylation and H3K27me3 deposition are mostly unaltered in the hybrid, with the exception of higher CHH DNA methylation in transposon-rich regions. However, A. thaliana genes enriched for the H3K27me3 mark are particularly likely to differ in expression between the hybrid and parent.

Conclusions: It has long been suspected that genome-scale properties cause the differential responses of genes from one or the other parent to hybridization. Our work links global chromatin compactness and H3K27me3 histone modification to global differences in gene expression in an interspecific Arabidopsis hybrid.
\end{abstract}

Keywords: Interspecific hybridization, Epigenome, Chromatin structure, Non-additive gene expression, Hi-C, Arabidopsis

\section{Background}

Interspecific hybridization is a common phenomenon in plants, as already recognized by Charles Darwin (reviewed in [1]). Hybrids are of interest to both evolutionary biologists and breeders, because they often show non-additive phenotypes, being either considerably more or less fit than the parents (reviewed in [2-7]). Although genetic distance clearly plays a role in dictating the extent of non-additive

\footnotetext{
*Correspondence: weigel@weigelworld.org; chang.liu@zmbp.unituebingen.de

†Equal contributors

'Department of Molecular Biology, Max Planck Institute for Developmental Biology, Tübingen 72076, Germany

Full list of author information is available at the end of the article
}

phenotypes, the relationship between whole-genome divergence and hybrid vigor or weakness is complex [8-11].

Interspecific hybridization can be regarded as an invasion of each parental genome by foreign genetic elements and this can lead to immediate and extensive genomic modifications [12-19]. "Transcriptome shocks," characterized by dramatic changes in gene expression, have been widely observed after interspecific hybridizations of plants and may contribute to the evolutionary success of emerging hybrids $[17,18,20,21]$. The changes in gene expression after hybridization have been, for example, attributed to either structural changes in the genome (e.g. resulting from loss of parental genomic fragments or mobilization of transposable 
elements) [16, 22, 23], complementation of recessive alleles [24-26], or epigenetic modifications [27-32]. In interspecific Arabidopsis hybrids, there can be striking asymmetry in expression changes of alleles derived from either parent, which has been linked to certain epigenetic chromatin marks [33, 34].

Interspecific hybridization and introgression are not uncommon in Arabidopsis [35] and several natural hybrids have been reported in this genus: $A$. suecica is an allopolyploid hybrid between $A$. thaliana and $A$. arenosa [36]; $A$. kamchatica is a hybrid between Siberian A. lyrata ssp. petraea and A. halleri [37, 38]; and extensive gene flow has been observed throughout the genus [39]. The existence of natural Arabidopsis hybrids stimulated early on work to understand genome-wide consequences of interspecific hybridization, including genome instability and gene expression, in such hybrids [13, 27, 40, 41]. One study reported that when genes were differentially expressed in an A. thaliana $\mathrm{x}$ lyrata hybrid, it was almost always biased towards higher expression of the A. lyrata allele [27]. Another study reported on $A$. thaliana x arenosa allopolyploids. Here, the vast majority of genes that were underexpressed in the hybrid relative to the mid-parental value were ones that were more highly expressed in the $A$. thaliana parent [13]. The molecular basis for these effects remains unknown.

Here, we relate genome-wide changes in gene expression in an A. thaliana x lyrata hybrid to changes in chromatin packing and epigenetic marks. We show that A. thaliana-derived genes are much more likely to change in expression than $A$. lyrata genes and that these are predominantly downregulated. Two important epigenetic marks, DNA methylation and H3K27me3, are mostly faithfully inherited in the $F_{1}$ hybrid, except for transposon-rich regions which acquired elevated $\mathrm{CHH}$ DNA methylation. In contrast, dramatic differences were seen in chromatin compactness, with the A. thaliana-derived chromosomes becoming much more compact in the hybrid. In addition, among all A. thaliana genes, those with H3K27me3 were most likely to vary in expression between $A$. thaliana and the hybrid.

\section{Results}

\section{Expression changes primarily in A. thaliana genes after} interspecific hybridization

We generated $A$. thaliana var. Col-0 x A. lyrata var. MN47 $F_{1}$ hybrid plants with $A$. thaliana as the maternal parent. A modified ovule rescue method [42] was applied to recover $F_{1}$ hybrid plants. As reported before for interspecific hybrids of $A$. thaliana and A. lyrata, the hybrid plants were larger than either parent before flowering, with leaf growth rate and leaf lamina color and thickness being more similar to A. lyrata $[42,43]$.

To compare transcriptomes in parents and hybrid, we performed RNA-sequencing (RNA-seq) analyses by mapping RNA-seq reads to a synthetic genome consisting of both the $A$. thaliana and A. lyrata reference genomes, retaining only uniquely mapped reads. We then assessed both relative expression of $A$. thaliana and $A$. lyrata orthologs in the hybrid, as well as expression of these genes compared with their corresponding parent. Consistent with an earlier report on hybrid plants derived from different $A$. thaliana and $A$. lyrata accessions as parents [27], there was a systematic shift towards $A$. lyrata alleles being more highly expressed in the hybrid than the corresponding A. thaliana alleles (Additional file 1: Figure S1A, $p<2.2 \times 10^{-16}$ with a Wilcoxon-Mann-Whitney test). However, the global expression profile of $A$. thaliana genes in the hybrid deviated much more from the $A$. thaliana parent than was the case for the A. lyrata genes (Fig. 1a and b). The higher expression stability of $A$. lyrata genes in the hybrid together with the higher overall expression levels likely explains the $A$. lyrata-like leaf morphology of the hybrid.

Compared with the $A$. thaliana parent, we identified 583 upregulated and 1233 downregulated $A$. thaliana genes in the hybrid; in contrast, only 156 and 55 genes from the $A$. lyrata genome were upregulated and downregulated, respectively (Fig. 1c and Additional file 2: Table S1). The upregulated or downregulated $A$. thaliana genes were distributed across all chromosomes (Additional file 1 : Figures $\mathrm{S} 1 \mathrm{~B}$ and $\mathrm{S} 1 \mathrm{C}$ ). That $A$. thaliana genes are much more likely to become downregulated than upregulated in

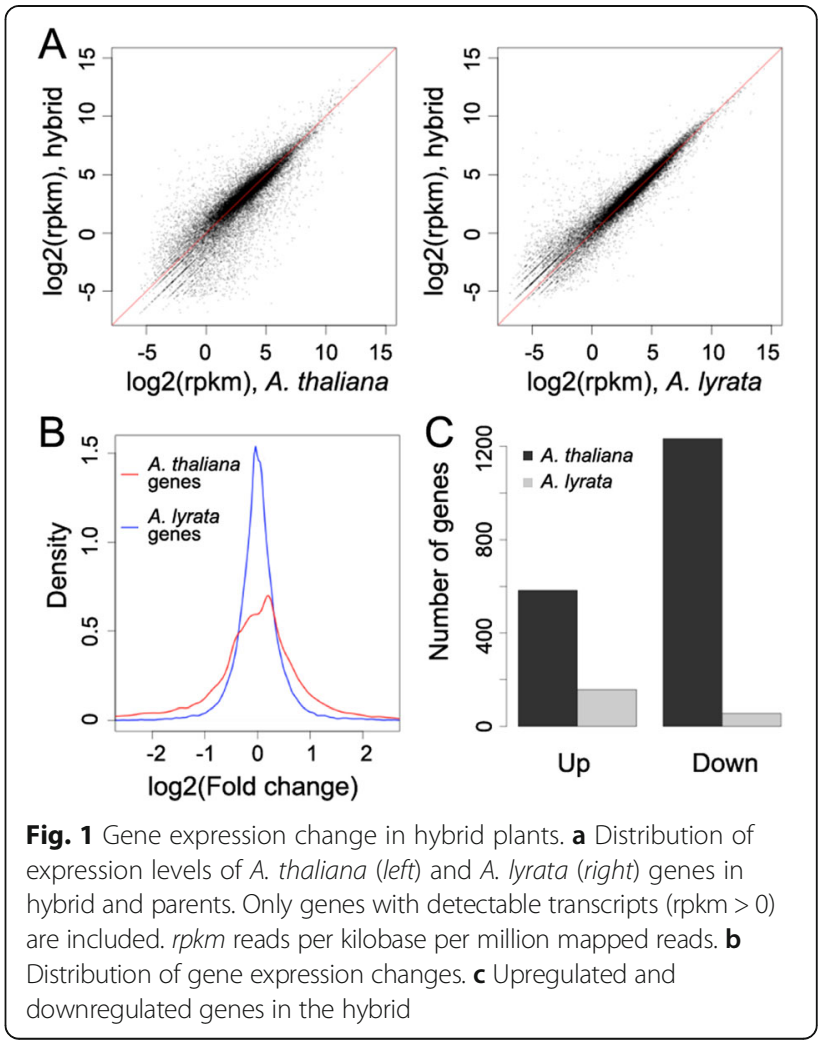


the hybrid has also been found in interspecific crosses between $A$. thaliana and $A$. arenosa, a close relative to $A$. lyrata [44], with hybrid plants resembling mostly the $A$. arenosa parent [13]. Thus, the altered expression specifically of the $A$. thaliana genes in interspecific hybrids appears to be a property of $A$. thaliana chromosomes [14].

Gene ontology (GO) analysis of the 1233 downregulated genes revealed a highly significant enrichment of genes associated with oxidative stress-response, metabolic processes, and cell wall organization, while upregulated genes were enriched for developmental processes related genes (Additional file 3: Table S2).

\section{Increased CHH methylation in the hybrid}

The systematically altered expression of $A$. thaliana genes in the hybrid suggested that this might be due to global epigenetic changes of the A. thaliana chromosomes. DNA methylation plays a pivotal role in regulating chromatin activities and can affect gene expression (reviewed in [45-47]). Due to a nearly fivefold higher TE density on the A. lyrata chromosome arms, they are on average much more methylated than those of $A$. thaliana [48, 49]. Global DNA methylation is often reprogrammed in hybrids, which can be viewed as an "epigenetic shock" from the combination of distinct parental epigenomes, even though changes of methylation in $F_{1}$ plants might not directly result in altered gene expression [50].

At a chromosomal level, we found that $\mathrm{CHH}$ methylation of the $A$. thaliana chromosomes increased in the hybrid, particularly in the centromeric and pericentromeric regions (Fig. 2a). The same regions showed only small increases in CHG methylation and CG methylation remained largely unchanged (Additional file 1: Figure S2). CHH methylation of the A. lyrata chromosomes was slightly increased in the hybrid, with a slight decrease of both CG and CHG methylation (Fig. 2a and Additional file 1: Figure S2). Although the A. lyrata centromeric regions were not accessible to analysis, adjacent regions experienced the largest increases in $\mathrm{CHH}$ methylation (Fig. 2), leading us to speculate that this trend might be even stronger for the pericentromeres themselves. The higher increment of $\mathrm{CHH}$ methylation around pericentromeres might be related to the higher density of TEs in these regions. In agreement, we found that $\mathrm{CHH}$ methylation increase correlated with TE density along the chromosome arms, indicating a TE-specific increase of $\mathrm{CHH}$ methylation in the hybrid (Fig. 2b and c).

$\mathrm{CHH}$ methylation at TEs is controlled by two partially overlapping pathways, with DRM1 (DOMAINS REARRANGED METHYLASE 1) and DRM2 being key factors responsible for $\mathrm{CHH}$ methylation and further contribution by an RdDM-independent CHROMOMETHYLASE
2 (CMT2)-dependent pathway [51-55]. Consistent with TEs in the pericentromere cores being mainly targeted by the RdDM-independent pathway (reviewed by [56]), A. thaliana genomic regions with elevated $\mathrm{CHH}$ methylation in the hybrid were those that have been reported to be preferentially sensitive to the loss of CMT2, but not DRM1/2 (Additional file 1: Figure S3A) [51, 52]. We observed a similar pattern, albeit with a weaker tendency, along the chromosome arms (Additional file 1: Figure S3B). Methylome changes, however, could apparently not explain the changes in expression of proteincoding genes in the hybrid (Fig. $2 \mathrm{~d}$ and Additional file 1: Figure S4).

\section{Increased compactness of $A$. thaliana chromatin in the hybrid}

DNA methylation is highly correlated with tightly packed heterochromatin (reviewed in [57]). The substantial increase of $\mathrm{CHH}$ methylation associated with the A. thaliana pericentromeric regions prompted us to ask whether the packing patterns of chromatin in hybrid and parents differed accordingly. To this end, we employed a genomewide Chromatin Conformation Capture approach, Hi-C [58], to compare chromatin packing. We adopted an in situ $\mathrm{Hi}-\mathrm{C}$ protocol that better preserves chromatin folding compared to the regular "dilution" Hi-C method [59-61]. After stringent read mapping and filtering, we obtained about 20 million, 38 million, and 85 million of true $\mathrm{Hi}-\mathrm{C}$ reads from $A$. thaliana, A. lyrata, and the hybrid, respectively (Additional file 4: Table S3). The normalized Hi-C maps showed strong signals along their diagonals, resulting from stochastic contacts between sequences close to each other in the linear genomes (Additional file 1: Figure S5). Visual inspection indicated that at a chromosomal level, the $A$. thaliana but not $A$. lyrata chromatin had more intra-chromosomal contacts over long distances in the hybrid (Fig. 3a and b). This pattern was confirmed by comparing the power-law decay curves of intrachromosomal interaction strength with genomic distance (Fig. 3a and b). Notably, both the pericentromeres and chromosome arms of $A$. thaliana showed less steep decay slopes in the hybrid compared to the parent (Fig. 3a). A possible scenario explaining these patterns might be that hybridization caused the $A$. thaliana chromosomes to become more compact and occupy smaller nuclear volumes, thereby increasing the likelihood and thus strength of long-distance chromatin interactions. To test this idea, we performed fluorescence in situ hybridization (FISH) with a pool of probes covering a 5.3-Mb genomic region (21.5 $\mathrm{Mb}$ to $26.8 \mathrm{Mb}$ ) in a non-pericentromeric region of A. thaliana chromosome 1 (see "Methods"). These probes did not produce a signal when hybridized to the A. lyrata genome (Fig. 3c). By calculating the volume of FISH signals (see "Methods"), we found that the signal occupied 


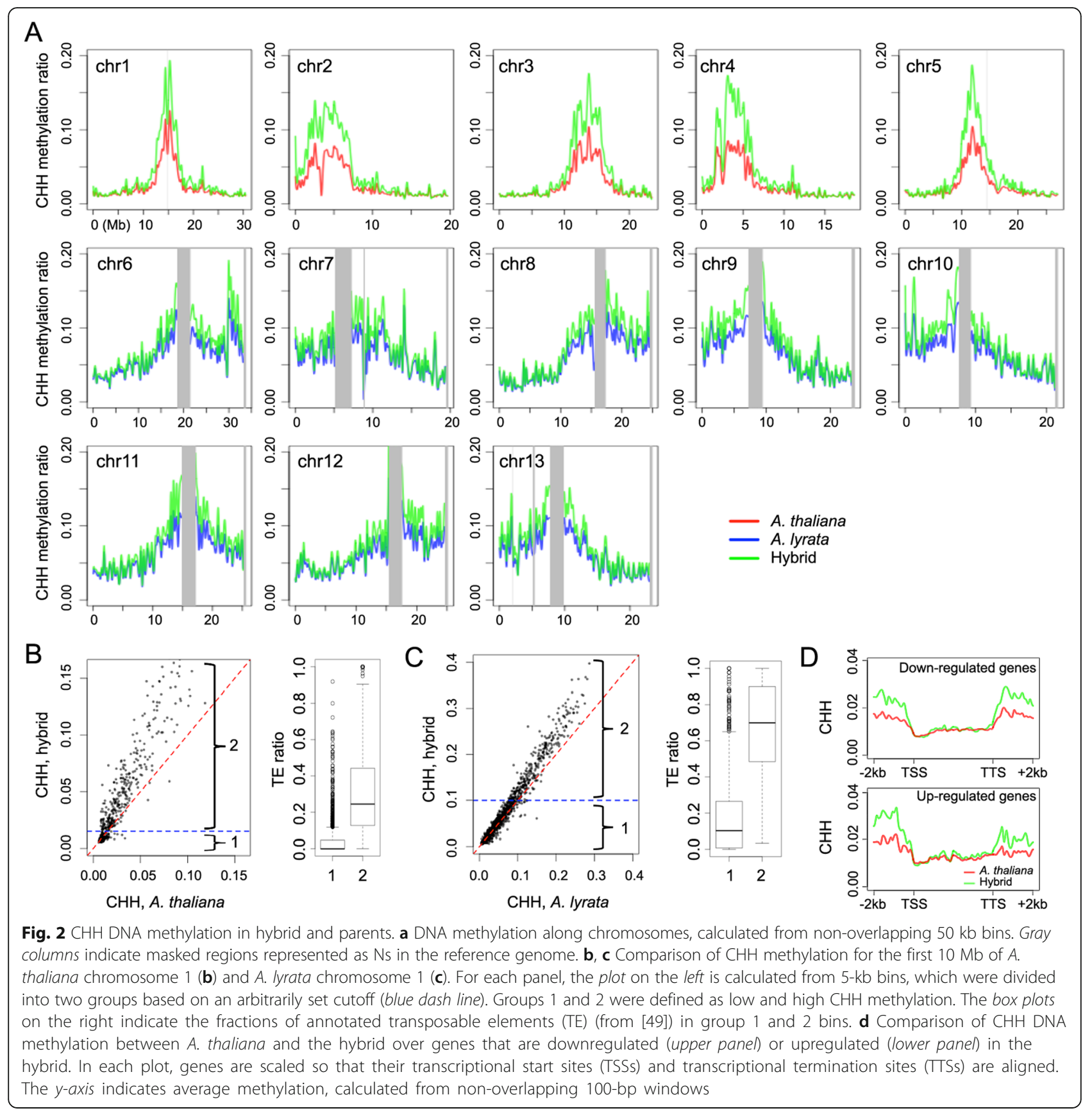

significantly less space in the hybrid nuclei than it did in the parent (Fig. 3d), indicating an increase in chromatin compactness of $A$. thaliana chromosomes in the hybrid.

Similarly, we observed a higher degree of $A$. thaliana chromatin compactness at local levels with $\mathrm{Hi}-\mathrm{C}$. By comparing the distribution of $\mathrm{Hi}-\mathrm{C}$ reads as a function of genomic distance, we found that compared to the parent, A. thaliana, but not A. lyrata, chromosomes in the hybrid engaged in more long-range interactions (Additional file 1: Figure S6). From Hi-C maps normalized at 5-kb resolution, we approximated the chromatin compactness of 5 -kb regions by calculating the strength of their interactions with surrounding regions located 10-50 kb away (see "Methods"). Overall, in the parents, chromatin was more compact in A. lyrata than in $A$. thaliana (Fig. 3e, compare red and blue boxplots). In the hybrid, the $A$. thaliana chromatin became more compact, whereas the A. lyrata chromatin became moderately less compact (Fig. 3e). Nonetheless, in the hybrid, A. lyrata chromatin still had higher compactness than $A$. thaliana chromatin (Fig. 3e, compare green boxplots). We next examined potential connections between DNA 

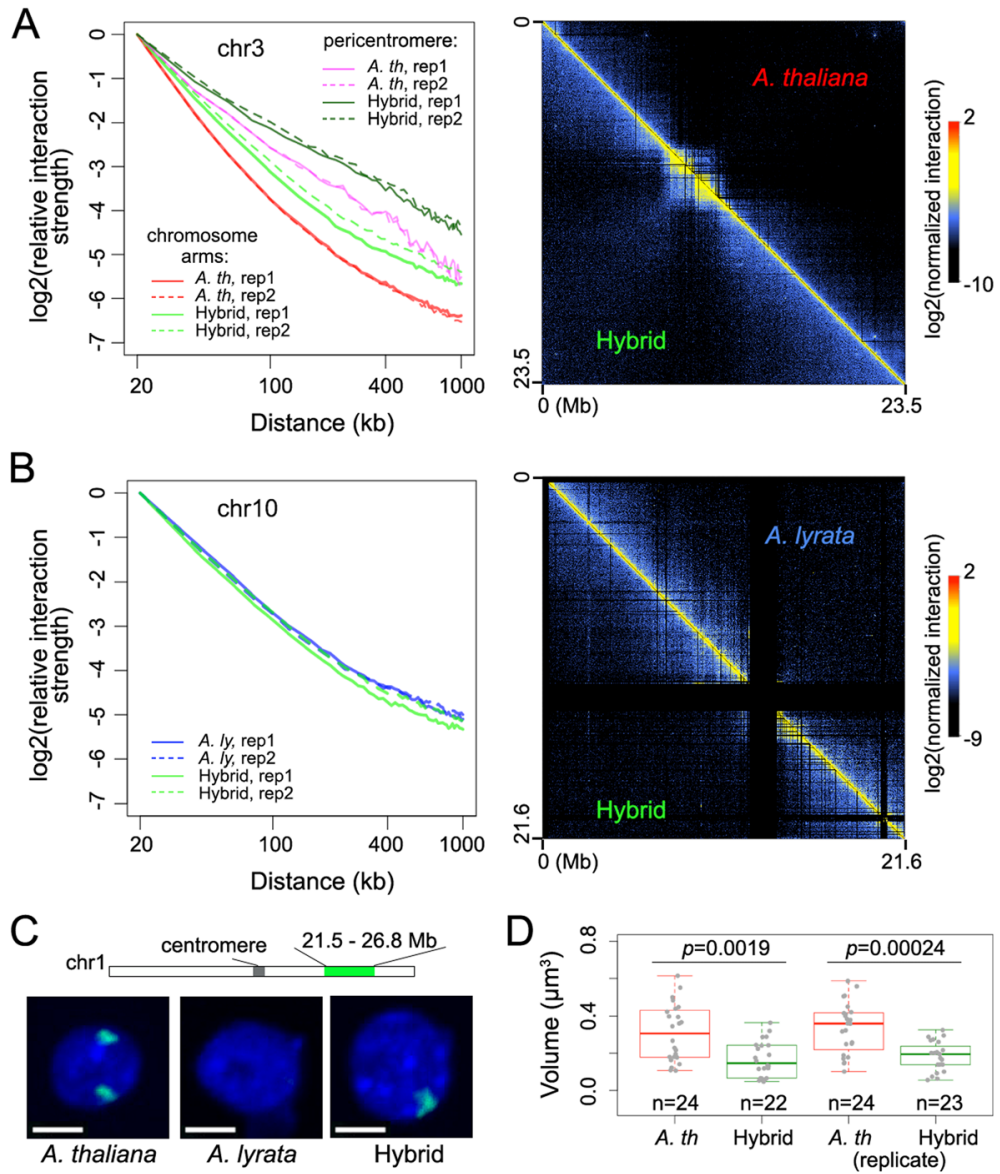

\section{$\mathrm{E}$}

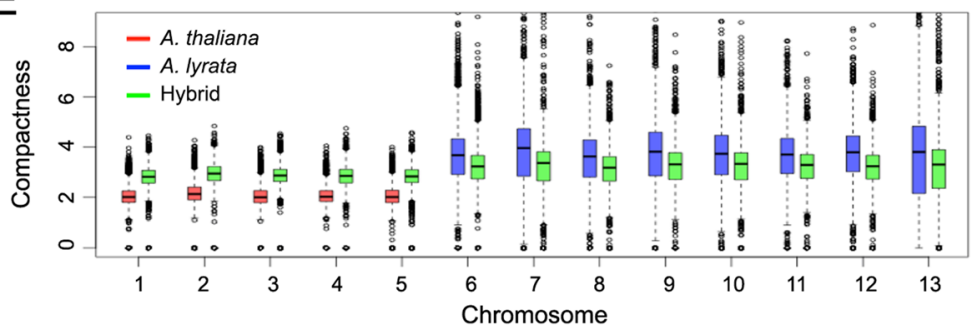

Fig. 3 Changes of chromosomal level chromatin compactness. a, b Comparisons of A. thaliana chromosome 3 and A. lyrata chromosome 5 (chromosome 10 in the synthetic hybrid reference genome) are shown in (a) and (b), respectively. For each panel, data from each biological replicate are shown on the left and the Hi-C maps from combined replicates are shown on the right. The plot on the left shows interaction decay exponents, calculated from $\mathrm{Hi}-\mathrm{C}$ maps at 20-kb resolution, which are shown on the right. For each curve, the contact frequency at 20-kb distance is set to 1. To make colors in the two Hi-C maps comparable, values in each normalized $\mathrm{Hi}-\mathrm{C}$ map were divided by the average value indicative of interactions between bins $20 \mathrm{~kb}$ apart. c, d FISH experiments. c A 5.3-Mb region (highlighted in green in the sketch) of the A. thaliana genome can be specifically detected with a BAC probe mixture. Scale bars, $2 \mu \mathrm{m}$. $\mathbf{d}$ Space volume occupied by the probed A. thaliana genomic region in the hybrid and the parent nuclei. $p$ values mean results of Wilcoxon-Mann-Whitney tests. e Analysis of chromatin compactness derived from $\mathrm{Hi}-\mathrm{C}$ maps at 5-kb resolution. Pericentromeric regions in $\mathrm{A}$. thaliana are excluded

methylation and chromatin packing in the hybrid and its parents. As expected, in all three genotypes, highly methylated chromatin regions had less negative interaction decay exponents (Additional file 1: Figure S7). The interaction decay exponents describe how fast chromatin interaction strengths drop with increasing genomic distance; as expected, heterochromatin, which is often tightly packed, has less negative values than euchromatin (see "Methods"). In the hybrid, $A$. thaliana chromatin was shifted towards less negative interaction decay exponents, making it more similar to A. lyrata chromatin (Additional file 1: Figure S7, see the last column). Overall, for all three types of plants, the more DNA methylation there is in a genomic region, the more highly compacted the chromatin is. 
Relationship between chromatin compactness, DNA methylation, and gene expression changes in the hybrid The chromatin packing effects were much more extensive than the DNA methylation changes, suggesting that altered DNA methylation is not the cause, or at least not the only cause, for changes in chromatin compaction. We used ratios of the $A$. thaliana chromatin compactness in the hybrid over its parent as an indicator of chromatin compaction. Selecting the top and bottom 10\% (Fig. 4a), we found that the regions with lowest compactness ratios tended to have higher DNA methylation in all sequence contexts (Fig. 4b, compare yellow and magenta box plots), which could not be explained by the differences of methylation between the hybrid and parents (Fig. 4b, compare box plots with the same color). Thus, the systematic increase of $A$. thaliana chromatin compactness in the hybrid was likely due to factor(s) other than DNA methylation.

Changes in chromatin compactness might affect its accessibility to transcription and chromatin remodeling factors, which could ultimately result in changes in gene expression levels [62, 63]. Given the fact that hybridization caused the $A$. thaliana chromatin to generally become more compact, we next assessed whether this was correlated with changes in the A. thaliana transcriptome (Fig. 1). Comparison of changes in gene expression in regions with the highest and lowest compactness ratios did not reveal any differences between the two classes (Fig. 4c). Similarly, we did not observe upregulated or downregulated genes to be more or less likely located in regions with low or high chromatin compactness ratios (Fig. $4 \mathrm{~d}$ and e). Thus, changes in $A$. thaliana chromatin compactness in the hybrid do not seem to be directly linked to changes in gene expression.

On the other hand, we observed that intra- and interchromosomal interactions among $A$. thaliana heterochromatin islands, known as KEEs or IHIs [64, 65], tended to be weaker in the hybrid (Additional file 1: Figure S8). Instead, KEE/IHI chromatin interacted more strongly with directly flanking sequences (Additional file 1: Figure S8B), which are more euchromatic [64]. We therefore asked whether this affected the transcriptional activities of KEE/ IHI regions, but found that genes located in KEEs/IHIs showed a similar profile of expression changes as the background (Additional file 1: Figure S8C). Collectively, our results suggest that after hybridization there is no close relationship between changes in chromatin compactness and gene expression on the A. thaliana chromosome arms.

\section{Enrichment of H3K27me3 in differentially expressed $A$. thaliana genes}

Next, we sought to analyze whether changes in expression of $A$. thaliana genes in the hybrid were associated
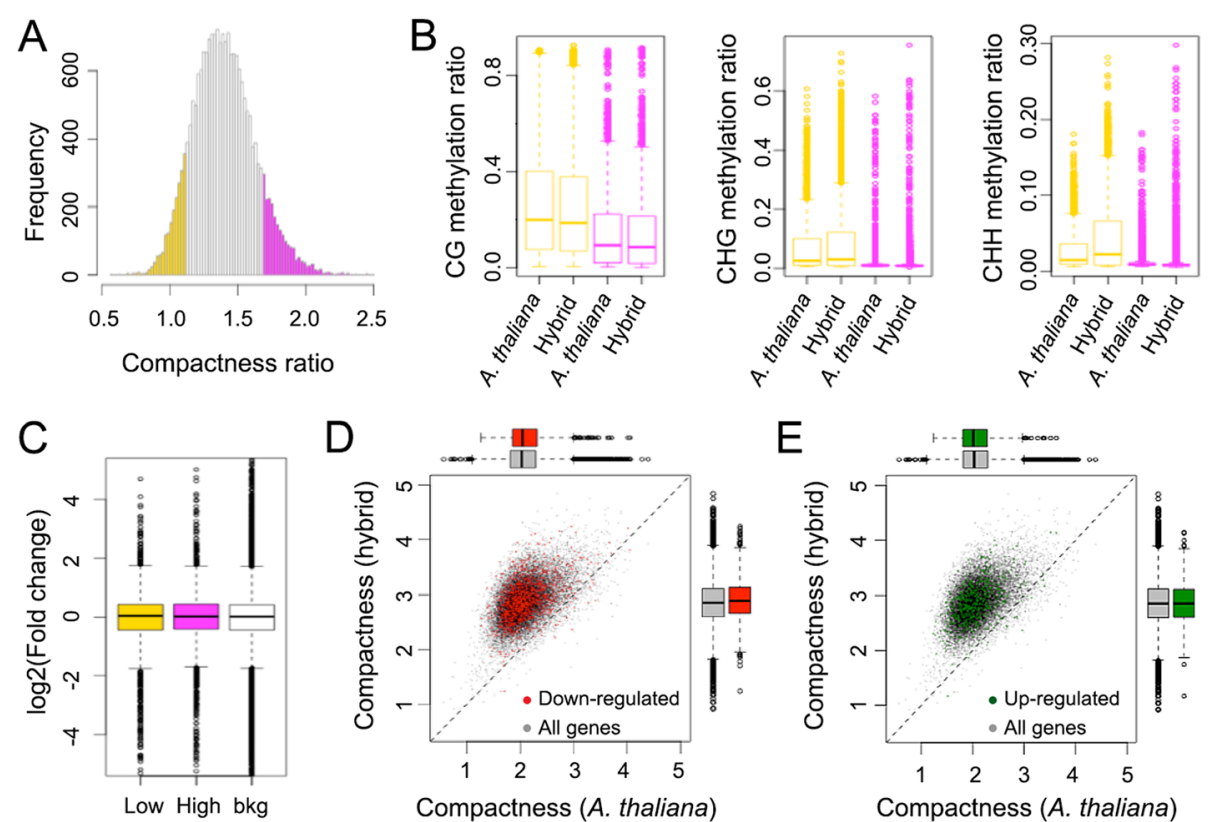

Fig. 4 Association between chromatin compactness, DNA methylation, and gene expression. a Distribution of $A$. thaliana chromatin compactness ratios between the hybrid and parent, calculated in 5-kb bins. Areas highlighted in yellow and magenta indicate the bottom and top $10 \%$ bins, respectively. $\mathbf{b}$ DNA methylation of bins highlighted in (a). c Distribution of gene expression ratios between hybrid and A. thaliana parent in regions highlighted in (a). Every gene is assigned to a 5-kb bin based on its TSS. Bkg background consisting of all genes. All comparisons have $p$ values $>0.05$ from Wilcoxon-Mann-Whitney tests. $\mathbf{d}$, e Relationship between chromatin compactness and differentially expressed genes. Bins of $5 \mathrm{~kb}$ are colored red (d) or green (e) if there is at least one upregulated or downregulated gene located in them. For both panels, all pairs of comparisons have $p$ values $>0.05$ from Wilcoxon-Mann-Whitney tests 
with genomic or epigenetic features of individual genes. Compared to the profile of all genes, upregulated or downregulated $A$. thaliana genes did not noticeably differ in length, exon number, and exon or intron size (Additional file 1: Figure S9). By making use of a previous analysis of the $A$. thaliana seedling epigenome based on a series of histone marks and variants [66], we found that both upregulated and downregulated $A$. thaliana genes could be differentiated from the genomic average of several marks in the parent, with the most prominent differences in H3K27me3, H3K36me3, H2A, and H2A.Z (Additional file 1: Figure S10). That the histone variant H2A.Z in gene bodies is associated with genes that are particularly sensitive to environmental or developmental changes $[67,68]$ agrees with our finding that such genes are highly enriched among the genes differentially expressed in the hybrid (Additional file 3: Table S2). An over-representation of $\mathrm{H} 3 \mathrm{~K} 27 \mathrm{me} 3$ in genes differentially expressed in the hybrid could simply be due to most of these genes being only weakly expressed in the A. thaliana parent, hence the increased H3K27me3 levels. This was indeed the case for genes upregulated in the hybrid (Fig. 5a). However, downregulated genes, which contributed the majority (68\%, see Fig. 1c) of differentially expressed $A$. thaliana genes, did not differ from the average in terms of expression level in the parent (Fig. 5a).

To understand why genes downregulated in the hybrid were enriched for the H3K27me3 mark, we compared the H3K27me3 landscape between parents and hybrid using ChIP-seq. We found highly similar H3K27me3 patterns in chromatin of hybrid and parents (Figs. 5b and c, Additional file 1: Figure S11), similar to observations made with intra-specific hybrids of $A$. thaliana $[69,70]$. There were a few exceptional loci with contrasting H3K27me3 levels in different genotypes, such as FLOWERING LOCUS C (FLC), which showed a complete loss of this mark throughout the coding region in the hybrid (Additional file 1: Figure S12). In this specific case, this was likely due to the presence of functional FRIGIDA (FRI) in the A. lyrata genome, activating FLC expression [71-73]. Most genes enriched for H3K27me3 in the parental genome maintained this mark in the hybrid (5130/ 6081 for A. thaliana genes and 5148/6671 for A. lyrata genes, Fig. 5d), indicating conservation of this histone mark at a global level upon hybridization. In line with our results derived from comparing differentially expressed genes with a variety of epigenetic marks (Additional file 1: Figure S10), genes marked with H3K27me3 in both the hybrid and $A$. thaliana were over-represented among genes downregulated in the hybrid (Fig. 5e). We wondered whether this downregulation was due to increased H3K27me3 deposition in the hybrid, but this was apparently not the case (Fig. 5f). Furthermore, compared to the total $A$. thaliana genes, as expected, genes that became upregulated in the hybrid showed a tendency of losing H3K27me3; however, downregulated genes did not show an increase in abundance of this histone mark in the hybrid (Fig. 5g).

\section{Increased expression variance of H3K27me3-marked $A$. thaliana genes in the hybrid}

The enrichment of the H3K27me3 mark among A. thaliana genes differentially expressed in the hybrid prompted us to further test whether on a genome-wide scale, $A$. thaliana genes labeled with H3K27me3 tended to have more transcriptional changes between the hybrid and parent. To this end, we analyzed genes enriched for H3K27me3 in both the hybrid and its parents (Additional file 5: Table S4). We observed parent-dependent changes of gene expression in the hybrid, with H3K27me3 marked A. thaliana genes, but not $A$. lyrata genes being downregulated (with $p$ values $<2.2 \times 10^{-16}$ and $=1.0$ from one-sided Wilcoxon-Mann-Whitney tests, respectively) (Fig. 6a). Moreover, on the A. thaliana side, compared to non-H3K27me3 target genes, we observed a larger variance of gene expression among genes marked with H3K27me3; however, this difference was not observed among A. lyrata genes (Fig. 6a). For both the A. thaliana and A. lyrata chromatin, the chromosomal H3K27me3 landscapes were similar between hybrid and parents (Fig. 5). Thus, being enriched for H3K27me3 per se was not sufficient to explain the differences in expression changes in the two subgenomes.

The repressive histone mark H3K27me3 has recently emerged as a key factor in regulating higher order chromatin organization in plants $[64,66,74-76]$. In A. thaliana, H3K27me3 is required for long-range chromatin interactions among certain loci [64] and is overrepresented in the promoter regions of genes that are in conformational linkage over long distances [66]. In both the hybrids and its parents, this histone mark was positively correlated with chromatin compactness (Fig. 6b and Additional file 1: Figure S13), suggesting that to a certain extent it is involved in spatial genome organization. Recent findings in animals indicate that transcriptional regulation of $\mathrm{H} 3 \mathrm{~K} 27 \mathrm{me} 3$-marked genes depends on contacts within three-dimensional chromatin networks [77-80]. At a genomic level, removal of Polycomb repression not only results in deregulation of gene expression, but also diminishes chromatin interactions among these loci $[77,80,81]$. In a case study of the Drosophila Hox genes, mutations on one PcG-targeted Hox gene weakens the silencing of other Hox genes that interact with it [79]. Thus, among H3K27me3 marked genes located in the $A$. thaliana genome, different from those located in the A. lyrata genome, the ones with increased variance of 

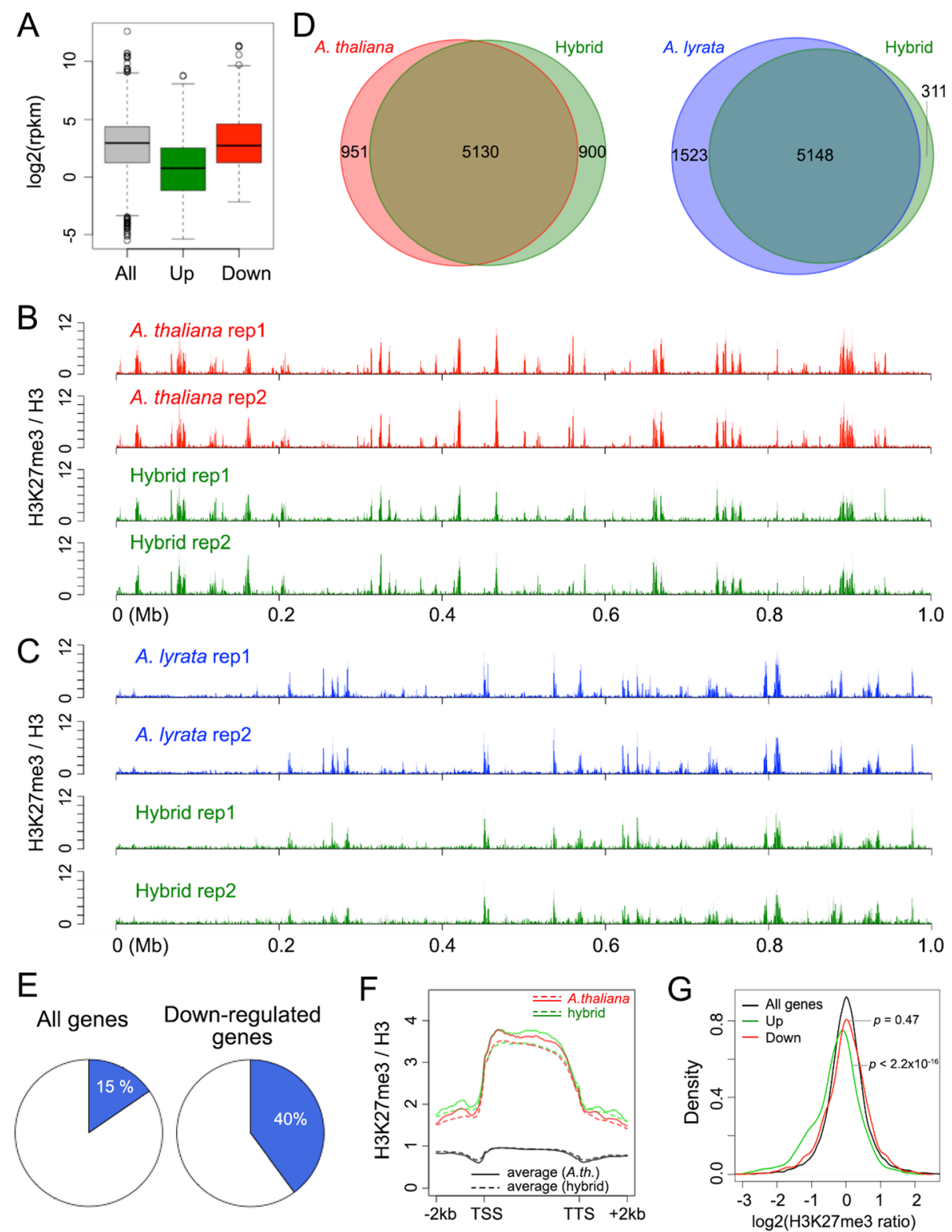

Fig. 5 H3K27me3-marked genes are enriched among genes downregulated in the hybrid. a Expression in A. thaliana of genes upregulated or downregulated in the hybrid. Green and red boxplots indicate genes upregulated and downregulated in the hybrid. b, c Distribution of H3K27me3 marks along a 1-Mb region on the A. thaliana chromosome $3(\mathbf{b})$ and the A. lyrata chromosome 5 (chromosome 10 in the synthetic hybrid reference genome) (c), plotted as the ratio of normalized coverage between H3K27me3 and H3 ChIP-seq with a 100-bp window setting. d Number of genes marked by H3K27me3 in different genotypes. A gene is considered marked with H3K27me3 if more than half of its transcribed region overlaps with H3K27me3 enrichment peaks. e Fraction of H3K27me3-marked genes shared by $A$. thaliana and the hybrid. The pie chart on the left refers to all $A$. thaliana genes, the one on the right to genes downregulated in the hybrid. Genes marked with H3K27me3 both in the hybrid and parent are colored blue. $\mathbf{f}$ Comparison of the H3K27me3 signals over A. thaliana gene bodies in hybrid and parent. For the parent, the red dashed curve indicates the average of all H3K27me3-marked genes and the red solid curve indicates the average of the subset downregulated in the hybrid and marked with H3K27me3 in both hybrid and parent. The green curves refer to the same sets in the hybrid. Black solid and dash curves indicate the average of all $A$. thaliana genes in the hybrid and parent. g Ratios of H3K27me3 signals in the hybrid over the parent in A. thaliana gene bodies. Upregulated or downregulated genes are the same as in (a). $p$ values mean Wilcoxon-Mann-Whitney tests

gene expression between the hybrid and parent might be linked to global genome compaction, which reflects changes in local chromatin folding topology.

\section{Discussion}

Gene expression studies of hybrids typically focus on additive versus non-additive gene expression of parent 


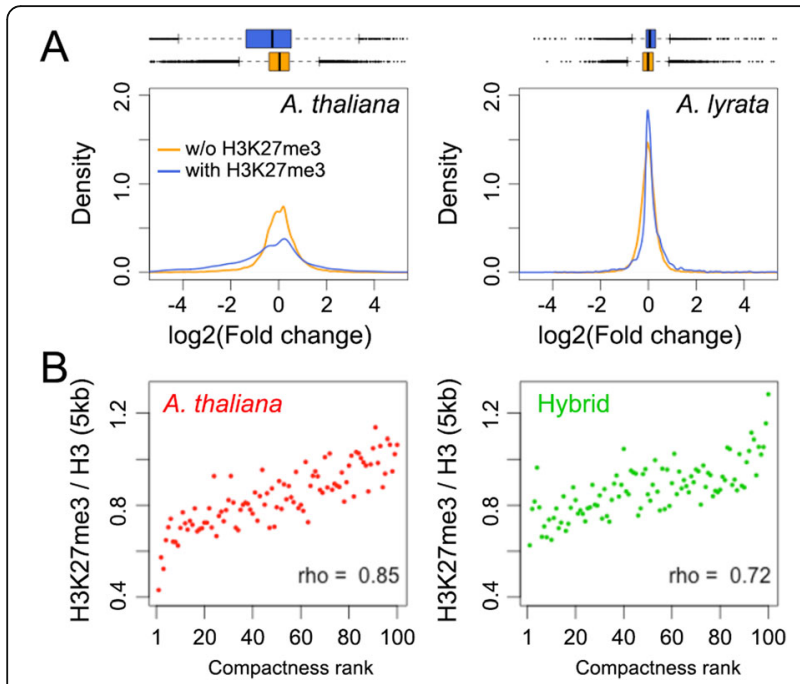

Fig. 6 Effects of hybridization on expression of H3K27me3-marked genes. a Distribution of $A$. thaliana (left) and A. lyrata (right) genes with respect to gene expression change in the hybrid. Only genes having similar H3K27me3 enrichment in parent and hybrid are included, where genes marked with H3K27me3 in both types of plants are colored in blue and genes not marked by H3K27me3 in either type of plant are colored orange. b Average H3K27me3 signals in A. thaliana chromatin in the hybrid and the parent ranked from weakest to strongest chromatin compactness

alleles, with an eye on understanding the phenomena of hybrid vigor and weakness (reviewed in [4, 82]). In this study, we focused instead on understanding how the transcriptomic readout of entire subgenomes in an interspecific hybrid differed from its parents. Our results revealed that genes differentially expressed between hybrid and parents were predominantly from the $A$. thaliana subgenome (Fig. 1). Similar to previous studies [27, 42], our attempts to generate hybrid plants with $A$. lyrata as the maternal parent were not successful. For this reason, one cannot assess how much maternal effects contribute to such transcriptome changes in hybrids. Nonetheless, our analyses point to differences in chromatin compactness and changes in chromatin compactness being an important driver of differential gene expression in the hybrid.

The mechanisms modulating higher-order chromatin organization in plants is poorly understood. It is known that chromatin regions with dense repressive epigenetic marks, such as DNA methylation and H3K27me3, are more tightly packed than those without such marks. For example, as the A. lyrata genome has more heterochromatic TE elements throughout its chromosomes [83], it is expected that $A$. lyrata chromatin is more compact than A. thaliana chromatin, both in the parent and the hybrid. Our Hi-C analyses show that this is indeed the case (Fig. 3e). However, the gain-of-compactness changes of $A$. thaliana chromosome arms in hybrids were not accompanied by increases in these repressive marks (Figs. 2 and 5). We do not know yet which factors caused this systematic change of chromatin compactness. For example, a dominant factor contributed by the A. lyrata genome might directly cause higher compaction of the $A$. thaliana genome. Also, we cannot exclude that having $A$. thaliana as the maternal parent plays a role in differential chromatin compaction. In the future, developing new culturing methods permitting reciprocal crosses between A. thaliana and A. lyrata can help address this question.

Generally, higher chromatin compactness is associated with lower gene expression [84], but such a correlation was not found at the level of individual genes (Figs. 4c-e). A possible explanation is that the average increase of $A$. thaliana chromatin compactness in the hybrid was not large enough to reach a tightly packed state comparable to inaccessible heterochromatin. As shown as an example in Fig. 3a, local compactness of $A$. thaliana chromosome 3 arms in the hybrid was still much lower than that of the pericentromeric regions in the parent. Nonetheless, genome-wide changes in chromatin compactness must ultimately be related to localized changes in chromatin torsion and tension, as well as chromatin conformation, all of which can influence DNA-protein and DNA-nucleosome interactions [85-89]. For these reasons, we speculate that there is a connection between the systematic changes in $A$. thaliana gene expression and chromatin compactness, although their causal relationship is not straightforward.

Genes labeled with the H3K27me3 histone mark were not only over-represented among differentially expressed A. thaliana genes (Fig. 5e and Additional file 1: Figure S10), but they also tended to have higher expression variance between hybrid and parents (Fig. 6a). Since the chromosome-scale H3K27me3 epigenomic landscapes of both the $A$. thaliana and A. lyrata genomes were basically unaltered in the hybrid (Fig. 5 and Additional file 1: Figure S11), one would like to know why $A$. thaliana H3K27me3 marked genes, but not A. lyrata H3K27me3 marked genes were affected in their expression in the hybrid. Because the H3K27me3 histone mark has an important role in the local recruitment of Polycomb group (PcG) proteins, it is highly relevant for long-range chromatin interactions. Several recent studies in animals have revealed that PcG proteins regulate gene expression in a three-dimensional network of chromatin contacts, where H3K27me3-marked chromatin regions that are distant along the chromosome tend to co-localize, to achieve coordinated transcriptional readouts of multiple genes [77-81]. In plants, genome-wide studies of chromatin packing have suggested that $\mathrm{H} 3 \mathrm{~K} 27 \mathrm{me} 3$, along with the associated PcG proteins, shapes genome structures [64-66, 75]. One example comes from the $F L C$ locus, where the two allelic copies cluster via H3K27me3-marked chromatin, 
with silencing mediated by PcG proteins [74]. On a genomic scale, loss of the LIKE HETEROCHROMATIN PROTEIN 1 (LHP1) protein, which binds to H3K27me3, results in alterations of both local H3K27me3 deposition and chromatin interaction patterns [76]. From a genome topology point of view, these findings have suggested that H3K27me3-targeted genes are similarly regulated in plants and animals. As discussed above, global changes in A. thaliana chromatin compactness were likely due to local conformational changes, which in turn may have positioned H3K27me3-marked genes into alternative regulatory environments.

Changes in compactness of the A. thaliana chromatin might also underlie nucleolar dominance, the selective silencing of ribosomal RNA (rRNA) genes contributed by one of the parental genomes in a hybrid [90]. In the A. thaliana x lyrata hybrid, the A. thaliana arrays of rRNA genes are specifically silenced [14, 91]. Being located in one of the two nucleolus organizer regions (NORs) is a prerequisite for rRNA gene silencing in the hybrid, since these genes become expressed when relocated to ectopic locations of the parental genome [91], indicating that nucleolar dominance is affected by the state of flanking chromatin. As one potential mechanism, it has been suggested that higher TE densities in regions adjacent to NOR2 (NOR ON CHROMOSOME 2) compared to those adjacent to NOR4 account for selective silencing of NOR2 rRNA genes [92]. Because short reads cannot be mapped to the highly repetitive NOR sequences, which in any case are not properly represented in the reference genome assemblies, we could not assess their behavior in the hybrid and parents, but we speculate that the $A$. thaliana NORs became more tightly packed and less accessible in the hybrid, as did both the $A$. thaliana chromosome arms and pericentromeric regions (Fig. 3). In A. thaliana nuclei, NORs preferentially interact with centromeres, which collectively form discrete foci of heterochromatin called chromocenters [93]. In the hybrid, the centromere-proximal boundaries of NOR2 and NOR4 interacted with pericentromeres as strongly as in A. thaliana (Additional file 1: Figure S14), suggesting maintenance of the NOR-centromere complexes. Chromocenters have been observed in A. thaliana x lyrata hybrid nuclei, appearing as conspicuous DAPI-dense spots [42]. From the higher levels of $\mathrm{CHH}$ methylation (Fig. 2) and higher chromatin compactness near centromeres in the hybrid (Fig. 3), it follows that if NORs are parts of chromocenters in the hybrid, they are likely in a more heterochromatic environment compared to the one in A. thaliana, with could contribute to selective rRNA gene silencing.

It is worth noting that chromatin compactness seems to be resistant to changes in nuclear morphology. In a recent $\mathrm{Hi}-\mathrm{C}$ study, neither the $A$. thaliana crowded nuclei 1 (crwn1) nor crwn4 mutant, which produce smaller and more spherical nuclei, showed evidence of increased chromatin compactness in chromosome arms $[65,94]$, suggestive of a mechanism actively modulating chromatin packing independently of the ratio of DNA and nuclear volume.

\section{Conclusions}

In conclusion, we report that $A$. thaliana-derived genes are much more likely to change in expression than $A$. lyrata-derived genes in an interspecific hybrid and that the differentially expressed $A$. thaliana genes tended to be labeled with the $\mathrm{H} 3 \mathrm{~K} 27 \mathrm{~m} 3$ histone mark. In addition, compared to the parent, A. thaliana chromatin compactness increases in the hybrid; while the compactness of A. lyrata chromatin hardly changes. By providing evidence for chromosome-scale changes of chromatin folding, we reveal a new mechanism that might underlie genome-wide differences in the behavior of two subgenomes in a hybrid.

\section{Methods \\ Plant material}

A. thaliana accession Columbia (Col-0) and A. lyrata accession MN47 [83] were used to generate hybrid plants, with $A$. thaliana as maternal parent. A modified ovule rescue method [42] was used to recover $F_{1}$ hybrid seeds. Six days after pollination, the elongating siliques were harvested and surface sterilized with $10 \%$ bleach for $20 \mathrm{~min}$ at room temperature. The siliques were opened under a dissecting microscope in a laminar flow cabinet and the developing seeds were transferred to half strength Murashige \& Skoog (MS) medium supplemented with $1 \%$ sucrose and $0.3 \%$ phytagel. The medium was placed in a standard long-day plant growth chamber $\left(23{ }^{\circ} \mathrm{C}\right.$, with $16 \mathrm{~h}$ light $/ 8 \mathrm{~h}$ dark cycles) to allow the hybrid seeds to mature and germinate. Upon germination, the hybrid seedlings were transferred to soil. The presence of the $A$. lyrata genome was verified by genotyping with a pair of A. lyrata specific primers, 5'CATAACTTTCGTTGTTACATC-3' and 5'-CCGAGTT ATTATGATTACTATTAGTC-3'.

For materials used in RNA-seq, bisulfite-sequencing (BS-seq), in situ Hi-C, and ChIP-seq experiments, the hybrid and the parents were grown at $23{ }^{\circ} \mathrm{C}$ in long days (16 h light/8 h dark) on soil. The aerial parts of 15 -dayold $A$. thaliana, 30-day-old A. lyrata, and hybrid seedlings, at which age the different genotypes had similar morphologies, were harvested at Zeitgeber time 6 (ZT6: $6 \mathrm{~h}$ after lights on). Note that differences in life history of the different samples might have contributed to observed expression and chromatin differences. However, because the growth rates and vegetative phase lengths of these plants are different, harvesting them at the same chronological time point likely leads to even stronger 
biases. For example, on day 10, the aerial portion of $A$. lyrata plants only includes very little true leaf material. On the other hand, on day 30 , the A. thaliana plants will already flower.

Two biological replicates were generated for each sample in all experiments. For BS-seq, $\mathrm{Hi}-\mathrm{C}$, and ChIP-seq, reads from replicates were combined. See Additional file 1: Figures S15-S17 for details of comparisons of replicates.

\section{RNA-seq library preparation and analysis}

Total RNA was isolated with RNeasy Plant Mini Kit (Qiagen) and libraries were prepared according to a standard protocol (Illumina). RNA-seq data from 15day-old Col-0 seedlings were from [95], in which plants were grown in the same growth chamber and with the same settings as used in our study. Although previous work from our lab has shown minimal expression differences in genetically identical plant cohorts grown at different times in our growth chambers [96], we cannot exclude that growth at different times contributes to the drastic differences in gene expression reported here. Reads were aligned against a synthetic reference genome, consisting of both the annotated A. thaliana (TAIR10) and A. lyrata genomes (v.1.0.24, ftp://ftp.ensemblgenomes.org/pub/plants/release-24/), using TopHat 2 with default parameters [97]. For the synthetic hybrid reference genome, the $A$. lyrata chromosomes 1 to 8 [83] were re-named as chromosomes 6 to 13. Only uniquely mapped reads were retained and processed with the GenomicAlignments package in $\mathrm{R}$ [98]. The resulting raw count table, which contained number of mapped reads for each gene in each sample, was used to identify differentially expressed genes with the DESeq2 package in $R$ [99]. We used criteria of false discover rate smaller than 0.05 and fold change of $\log 2$ fold greater than 2 to call upregulated and downregulated genes. Gene ontology (GO) analysis of differentially expressed $A$. thaliana alleles was performed according to [100], where the enriched GO terms were identified with GOrilla [101] and further summarized with REViGO [102]. In parallel, this count table was also used to calculate RPKM (Reads Per Kilobase per Million mapped reads) for each gene [103].

\section{In situ Hi-C library preparation}

Tissue fixation and nuclei extraction were performed as described [75]. For one round of in situ Hi-C library preparation, $0.5 \mathrm{~g}$ of homogenized tissue powder produced via grinding fixed tissue under liquid nitrogen was used.

Nuclei permeabilization, chromatin digestion, and proximity ligation treatments were adapted from [60]. The extracted nuclei were resuspended in $150 \mu \mathrm{L} 0.5 \%$ SDS and split equally into three tubes. To make the nuclei more permeable, the resuspended nuclei were incubated at $62{ }^{\circ} \mathrm{C}$ for $5 \mathrm{~min}$, after which $145 \mu \mathrm{L}$ water and $25 \mu \mathrm{L} 10 \%$ Triton X-100 were added and incubated at $37^{\circ} \mathrm{C}$ for $15 \mathrm{~min}$. Next, the nuclei in each tube were digested by adding $25 \mu \mathrm{L}$ 10x NEB buffer $3(100 \mathrm{mM}$ $\mathrm{NaCl}, 50 \mathrm{mM}$ Tris- $\mathrm{HCl}, 10 \mathrm{mM} \mathrm{MgCl}, 1 \mathrm{mM}$ DTT, $\mathrm{pH}$ 7.9) and $50 \mathrm{U}$ of DpnII restriction enzyme and incubated at $37{ }^{\circ} \mathrm{C}$ overnight. The next day, the nuclei were incubated at $62{ }^{\circ} \mathrm{C}$ for $20 \mathrm{~min}$ to inactivate the restriction enzyme. Next, the digested chromatin was bluntended by adding $1 \mu \mathrm{L}$ of $10 \mathrm{mM}$ dTTP, $1 \mu \mathrm{L}$ of $10 \mathrm{mM}$ dATP, $1 \mu \mathrm{L}$ of $10 \mathrm{mM}$ dGTP, $25 \mu \mathrm{L}$ of $0.4 \mathrm{mM}$ biotin14-dCTP, $14 \mu \mathrm{L}$ water, and $4 \mu \mathrm{L}(40 \mathrm{U})$, Klenow fragment and incubated at $37{ }^{\circ} \mathrm{C}$ for $2 \mathrm{~h}$. Subsequently, $663 \mu \mathrm{L}$ water, $120 \mu \mathrm{L} 10 \mathrm{x}$ blunt-end ligation buffer (300 mM Tris- $\mathrm{HCl}, 100 \mathrm{mM} \mathrm{MgCl} 2,100 \mathrm{mM}$ DTT, $1 \mathrm{mM}$ ATP, pH 7.8), $100 \mu \mathrm{L} 10 \%$ Triton X-100, and 20 Weiss U T4 DNA ligase were added to start proximity ligation. The ligation reaction was placed at room temperature for $4 \mathrm{~h}$. After ligation, the nuclei were collected by spinning them down at $1000 \mathrm{rcf}$ for $3 \mathrm{~min}$ and then resuspended in $750 \mu \mathrm{L}$ SDS buffer $(50 \mathrm{mM}$ Tris$\mathrm{HCl}, 1 \%$ SDS, $10 \mathrm{mM}$ EDTA, $\mathrm{pH}$ 8.0) and incubated with $200 \mu \mathrm{g}$ proteinase $\mathrm{K}$ at $55{ }^{\circ} \mathrm{C}$ for $30 \mathrm{~min}$. The formaldehyde crosslink was reversed by adding $30 \mu \mathrm{L} 5 \mathrm{M}$ $\mathrm{NaCl}$ to the solution followed by overnight incubation at $65{ }^{\circ} \mathrm{C}$. The recovery of Hi-C DNA and subsequent DNA manipulations were performed as described [75]. The final library was sequenced on an Illumina HiSeq 3000 instrument with $2 \times 150$ bp reads.

\section{$\mathrm{Hi}-\mathrm{C}$ read mapping and filtering}

$\mathrm{Hi}-\mathrm{C}$ reads from both hybrid and parents were mapped to the synthetic hybrid genome assembly as described above for RNA-seq. With an iterative mapping strategy [66], the 5' $100 \mathrm{bp}$ sequences of reads 1 and 2 of the mate pairs were mapped independently and only pairedend reads with both mates uniquely mapped were kept. Removal of polymerase chain reaction (PCR) duplicates and read filtering were performed as described [66], except that the hybrid reference genome was used. Hi-C reads from each biological replicate used in this study are summarized in Additional file 4: Table S3.

\section{Hi-C map normalization}

For normalization, a count matrix was firstly created and filled with all filtered $\mathrm{Hi}-\mathrm{C}$ reads with respect to their genomic positions. An iterative bias correction method was employed to account for technical biases, such as PCR amplification efficiency, restriction sites density, and mapping biases [104]. Under an assumption that each bin should have equal "visibility" (sequence coverage) in a $\mathrm{Hi}-\mathrm{C}$ experiment, the bias correction process adjusted the count matrix so that at the end 
each row or column of the $\mathrm{Hi}-\mathrm{C}$ matrix had similar sum values [105]. To this end, an iterative matrix correction function in the "HiTC" package in R was used [106]. We stopped the iterative correction loop when the eps value, which was used as a measurement of how similar the matrices in two consecutive correction steps were, dropped below $1 \times 10^{-4}$. Normalization at $20-\mathrm{kb}$ resolution was done at a genome-wide level (i.e. all chromosomes were included), while normalization at $5-\mathrm{kb}$ resolution was done for each chromosome separately. In the 20-kb resolution Hi-C map of the hybrid, we observed signals of interactions between chromatin from the two parents that were highly correlated with the distribution of syntenic blocks of the two genomes [83] (Additional file 1: Figure S5C). These signals were not discussed in this study, as our analyses indicated that mapping errors were at least partially responsible for these patterns (Additional file 1: Figures S18 and S19). The overall impact of reads mapping errors on calculating interaction decay exponents was negligible (see below).

\section{Calculation of chromatin compactness and interaction decay exponents}

To calculate chromatin compactness (Fig. 3e), the entries in a normalized $\mathrm{Hi}-\mathrm{C}$ matrix (5-kb resolution) were first divided by the average value of entries, resulting in measurements of neighboring bin interactions. Such scaling procedure offset differences between $\mathrm{Hi}-\mathrm{C}$ matrices due to different sequencing depths. For each $5-\mathrm{kb}$ bin, its chromatin compactness was defined as the sum of its interactions with all bins located 10-50 kb away.

The interaction decay exponent of 100-kb genomic regions (Additional file 1: Figure S7) was calculated as follows: a subset of the normalized Hi-C matrix $(5 \mathrm{~kb})$ corresponding to a given 100-kb region was extracted and the average value of entries indicating chromatin interaction strengths of 5-kb, 10-kb, 15-kb, ..., 50-kb distances was calculated accordingly. Because entries associated with bins masked in the Hi-C matrix normalization step were excluded, at the end, there might be fewer than ten average values. We only continued with regions that generated at least six average values, which we used subsequently for linear regression with the "Im" function in R. The resulting slope was defined as the interaction decay exponent.

Because both the $A$. thaliana and A. lyrata genomes showed a genome-wide even error rate in read mapping (Additional file 1: Figures S18B and S19B), during the calculation of the interaction decay exponents and after logarithm transformation, the error rate became a constant that only changed the y-axis intercept, but not its slope.

\section{Bisulfite sequencing and data analysis}

Genomic DNA was extracted from leaves of hybrid and parent seedlings with the DNAeasy Plant Mini
Kit (Qiagen). DNA was sheared to 350 bp with a Covaris $^{\text {tw }}$ S220 sonicator. DNA end repair, A-tailing, and adaptor ligation were performed with a TruSeq Nano Kit (Illumina) according to the manufacturer's instructions. After adaptor ligation, the bisulfite treatment was done with an Epitect Plus DNA Bisulfite Conversion Kit (Qiagen). PCR amplification of library molecules was done using KAPA Hifi Uracil + DNA Polymerase (Kapa Biosystems). Libraries were sequenced on an Illumina HiSeq 3000 instrument with $2 \times 150$ bp reads.

All paired-end reads were aligned to the synthetic hybrid reference genome using Bismark (v0.15.0) [107] with Bowtie 2 aligner (v2.2.4) [108] with default parameter settings. PCR duplicates were removed after mapping. The unmethylated and methylated cytosine residue(s) in every read were identified in all sequence contexts (CG, CHG, and $\mathrm{CHH}$ ) by the "bismark_methylation_extractor" script in Bismark. The methylation ratio of a given genomic region was calculated as the ratio between the total number of identified 5-methylcytosines and the total number of sequenced cytosines.

\section{ChIP-seq and data analysis}

Tissue fixation and nuclei extraction were performed according to [75] and nuclei from $1 \mathrm{~g}$ of seedlings were used for one round of ChIP. The ChIP experiments essentially followed [66] with minor changes. In brief, chromatin was sheared to an average size of $350 \mathrm{bp}$ with a Covaris E220evolution ${ }^{\text {Th }}$ sonicator. The sonicated sample was halved and immunoprecipitated with $2 \mu \mathrm{g}$ of anti-H3 (Abcam ab1791) or $2 \mu \mathrm{g}$ of anti-H3K27me3 antibodies (Millipore, 07-449). After overnight incubation at $4{ }^{\circ} \mathrm{C}$, the antibodies were recovered with $15 \mu \mathrm{L}$ Protein A/G magnetic beads (Pierce) followed by a series of washing steps as described [66]. The ChIP-ed DNA was extracted with a standard phenol-chloroform method and the subsequent end repairing, A-tailing, adaptor ligation, and library amplification steps were performed with the NEBNext $^{\circ}$ Ultra $^{\text {Tx }}$ II DNA Library Prep Kit (NEB). The library was sequenced on an Illumina HiSeq 3000 instrument with $2 \times 150$ bp reads.

Reads were aligned against the synthetic hybrid reference genome, as described above, using Bowtie 2 v2.2.4 [108] with mapping parameters as "-R 5 - $\mathrm{N} 0$ - 020 -i S,1,0.50." The mapped reads were analyzed by MACS2 v2.1.0.20140616 [109]. The "-broad" flag was on during peak calling, with reads from the anti-H3 sample used as control and default settings were used for the other parameters.

\section{FISH}

FISH probes were labeled with digoxigenin-11-dUTP by nick translation. A collection of bacterial artificial 
chromosome (BAC) cloning containing A. thaliana genomic fragments belonging to a 5.3-Mb interval on the right arm of chromosome 1 were used (Additional file 6: Table S5). The final probe concentration was $50 \mathrm{ng} / \mu \mathrm{L}$.

Nuclei were isolated from fixed plant tissue, stained with DAPI, and sorted with a MoFlo ${ }^{\mathrm{Tm}}$ XDP FACS instrument (Beckman Coulter) using a 100- $\mu$ m CytoNozzle and standard PBS as sheath. Nuclei were sorted in Purify 1-drop mode. DAPI was excited using an OBIS $375 \mathrm{~nm}$ LX laser at $40 \mathrm{~mW}$ focused using a Near UV achromatic lens (Newport PAC14AR.15) with scatter collected from a 488-nm Argon $(70 \mathrm{~mW})$ and elliptically focused. Peak DAPI was collected in FL8 (405/30) and FL9 (465/30), with the shoulder in FL10 (542/27). Events were triggered off FL8 and nuclei were first identified by SSC-LA versus FL9-LA. Clumps were removed by sequential plotting of SSC-W, FSC-W, FL8-W versus FL8-LH with further auto-fluorescent debris removed by plotting FL8LH versus FL10-LH. Finally, fully gated nuclei with different ploidy levels were inferred according to their increasing DAPI signal intensities in a bivariate FL8-LH versus SSC-LA plot; only the $2 \mathrm{C}$ nuclei were collected. The nuclei were then transferred onto a glass slide. Next, the pre-hybridization, hybridization, post-hybridization wash, and probe detection steps were performed as described with minor modifications [110, 111], with hybridization carried out at $45^{\circ} \mathrm{C}$ for $20 \mathrm{~h}$. After the final wash step, slides were mounted with SlowFade ${ }^{\circ}$ Diamond Antifade Mountant with DAPI (Thermo Fisher Scientific).

\section{Fluorescence microscopy and image processing}

Confocal images were acquired with a Zeiss LSM 880 Airyscan system. For each nucleus, z-stack images (with $0.22 \mu \mathrm{m}$ thickness for each optical sectioning) were taken with a $63 \times$ objective lens. The detection of DAPI and Alexa Fluor 488 was according to the following settings: laser power $=1.3 \%$ for $405 \mathrm{~nm}$ and $0.67 \%$ for $488 \mathrm{~nm}$; pinhole = 1 AU; filter $=$ BP 420-480 + BP 495-550; master gain $=700$; digital gain $=2$. Same parameter settings were applied to all types of nuclei for image acquisition. Because each $A$. thaliana nucleus has two copies of target genomic DNA, it is not possible to determine the volume occupied by each copy if they overlap in space. Thus, for ease of image processing, we only recorded images of $A$. thaliana nuclei displaying two distinct clusters of FISH signals. Image processing was done with Fiji software and images were finally assembled in Photoshop. The volumes occupied by the probed genomic region in different nuclei were approximated according to the sum of areas of filtered FISH signals in z-stack images (Additional file 1: Figure S20). To identify pixels with FISH signal in each zstack image, a threshold value of 25 in the green channel was used throughout.

\section{Additional files}

Additional file 1: Figures S1-S20. Supplemental references and
Supplemental Figures S1-S20. (DOCX $13462 \mathrm{~kb}$ )

Additional file 2: Table S1. RNA-seq analysis, including reads counting, statistical analysis, on gene expression changes. (XLSX 11493 kb)

Additional file 3: Table S2. GO analysis of differentially expressed genes. (XLSX $82 \mathrm{~kb}$ )

Additional file 4: Table S3. Statistics of Hi-C reads. (XLSX 9 kb)

Additional file 5: Table S4. Genes enriched for H3K27me3 marks. (XLSX 3496 kb)

Additional file 6: Table S5. BAC vectors used for making FISH probes. (XLSX 44 kb)

\section{Acknowledgements}

We thank Christa Lanz, Julia Hildebrandt, and Katrin Fritschi from the Max Planck Institute for Developmental Biology for assistance with sequencing. We thank Monika Demar and Sonja Kersten for technical assistance. We thank members of the Liu and Weigel laboratories for critical review and comments on the manuscript.

\section{Funding}

This work was supported by Marie Curie Fellowship PIIF-GA-2012-327608 (CL), Deutsche Forschungsgemeinschaft LI 2862/1 (CL), a grant from the DFG Collaborative Research Center SFB1101 (DW), ERC AdG IMMUNEMESIS (DW), and the Max Planck Society (DW).

Availability of data and materials

RNA-seq, in situ Hi-C, BS-seq, and ChIP-seq short read data have been submitted to the NCBI Sequence Read Archive (SRA; http://

www.ncbi.nlm.nih.gov/sra) under accession number SRP095993.

\section{Authors' contributions}

$\mathrm{CL}$ and DW conceived and designed the experiments. CL conducted $\mathrm{Hi}-\mathrm{C}$ and RNA-seq experiments. CB conducted BS-seq experiments. WZ conducted ChIP-seq experiments. BH conducted FISH experiments. KWB and ESD performed the FACS experiment. WZ, CL, and DW analyzed and interpreted the data. All authors wrote the paper and approved the final manuscript.

Ethics approval and consent to participate

Not applicable.

Competing interests

The authors declare that they have no competing interests.

\section{Publisher's Note}

Springer Nature remains neutral with regard to jurisdictional claims in published maps and institutional affiliations.

\section{Author details}

${ }^{1}$ Department of Molecular Biology, Max Planck Institute for Developmental Biology, Tübingen 72076, Germany. ${ }^{2}$ Center for Plant Molecular Biology (ZMBP), University of Tübingen, Auf der Morgenstelle 32, Tübingen 72076, Germany. ${ }^{3}$ Present Address: Gregor Mendel Institute, Austrian Academy of Sciences, Dr. Bohr-Gasse 3, A-1030, Vienna, Austria.

Received: 4 June 2017 Accepted: 18 July 2017

Published online: 22 August 2017

\section{References}

1. Mallet J. Hybridization as an invasion of the genome. Trends Ecol Evol. 2005; 20:229-37.

2. Baack EJ, Rieseberg LH. A genomic view of introgression and hybrid speciation. Curr Opin Genet Dev. 2007;17:513-8.

3. Chen ZJ. Molecular mechanisms of polyploidy and hybrid vigor. Trends Plant Sci. 2010;15:57-71.

4. Chen ZJ. Genomic and epigenetic insights into the molecular bases of heterosis. Nat Rev Genet. 2013;14:471-82. 
5. Fridman E. Consequences of hybridization and heterozygosity on plant vigor and phenotypic stability. Plant Sci. 2015;232:35-40.

6. Schnable PS, Springer NM. Progress toward understanding heterosis in crop plants. Annu Rev Plant Biol. 2013;64:71-88.

7. Hochholdinger F, Hoecker $\mathrm{N}$. Towards the molecular basis of heterosis. Trends Plant Sci. 2007;12:427-32.

8. Meyer RC, Torjek O, Becher M, Altmann T. Heterosis of biomass production in Arabidopsis. Establishment during early development. Plant Physiol. 2004; 134:1813-23.

9. Cerna FJ, Cianzio SR, Rafalski A, Tingey S, Dyer D. Relationship between seed yield heterosis and molecular marker heterozygosity in soybean. Theoretical and Applied Genetics. 1997;95:460-7.

10. Liu ZQ, Pei Y, Pu ZJ. Relationship between hybrid performance and genetic diversity based on RAPD markers in wheat, Triticum aestivum L. Plant Breeding. 1999;118:119-23.

11. Riday H, Brummer EC, Campbell TA, Luth D, Cazcarro PM. Comparisons of genetic and morphological distance with heterosis between Medicago sativa subsp. sativa and subsp. falcata. Euphytica. 2003;131:37-45.

12. Yoo MJ, Szadkowski E, Wendel JF. Homoeolog expression bias and expression level dominance in allopolyploid cotton. Heredity (Edinb). 2013; 110:171-80.

13. Wang J, Tian L, Lee HS, Wei NE, Jiang $H$, Watson $B$, et al. Genomewide nonadditive gene regulation in Arabidopsis allotetraploids. Genetics. 2006; 172:507-17.

14. Beaulieu J, Jean M, Belzile F. The allotetraploid Arabidopsis thalianaArabidopsis lyrata subsp. petraea as an alternative model system for the study of polyploidy in plants. Mol Genet Genomics. 2009;281:421-35.

15. Hegarty MJ, Barker GL, Brennan AC, Edwards KJ, Abbott RJ, Hiscock SJ, Changes to gene expression associated with hybrid speciation in plants: further insights from transcriptomic studies in Senecio. Philos Trans R Soc Lond B Biol Sci. 2008;363:3055-69.

16. Doyle JJ, Flagel LE, Paterson AH, Rapp RA, Soltis DE, Soltis PS, et al. Evolutionary genetics of genome merger and doubling in plants. Annu Rev Genet. 2008:42:443-61.

17. Hegarty MJ, Barker GL, Wilson ID, Abbott RJ, Edwards KJ, Hiscock SJ. Transcriptome shock after interspecific hybridization in senecio is ameliorated by genome duplication. Curr Biol. 2006;16:1652-9.

18. Abbott R, Albach D, Ansell S, Arntzen JW, Baird SJ, Bierne N, et al. Hybridization and speciation. J Evol Biol. 2013;26:229-46.

19. Chen ZJ. Genetic and epigenetic mechanisms for gene expression and phenotypic variation in plant polyploids. Annu Rev Plant Biol. 2007;58:377-406.

20. McClintock B. The significance of responses of the genome to challenge. Science. 1984;226:792-801.

21. Buggs RJ, Zhang L, Miles N, Tate JA, Gao L, Wei W, et al. Transcriptomic shock generates evolutionary novelty in a newly formed, natural allopolyploid plant. Curr Biol. 2011;21:551-6.

22. Feldman M, Liu B, Segal G, Abbo S, Levy AA, Vega JM. Rapid elimination of low-copy DNA sequences in polyploid wheat: a possible mechanism for differentiation of homoeologous chromosomes. Genetics. 1997;147:1381-7.

23. Song K, Lu P, Tang K, Osborn TC. Rapid genome change in synthetic polyploids of Brassica and its implications for polyploid evolution. Proc Natl Acad Sci U S A. 1995;92:7719-23.

24. Birchler JA, Yao H, Chudalayandi S, Vaiman D, Veitia RA. Heterosis. Plant Cell. 2010;22:2105-12.

25. Paschold A, Jia Y, Marcon C, Lund S, Larson NB, Yeh CT, et al. Complementation contributes to transcriptome complexity in maize (Zea mays L.) hybrids relative to their inbred parents. Genome Res. 2012;22:2445-54.

26. Springer NM, Stupar RM. Allelic variation and heterosis in maize: how do two halves make more than a whole? Genome Res. 2007;17:264-75.

27. He F, Zhang X, Hu J, Turck F, Dong X, Goebel U, et al. Genome-wide analysis of cis-regulatory divergence between species in the Arabidopsis genus. Mol Biol Evol. 2012;29:3385-95.

28. Lee HS, Chen ZJ. Protein-coding genes are epigenetically regulated in Arabidopsis polyploids. Proc Natl Acad Sci U S A. 2001;98:6753-8.

29. Wang J, Tian L, Madlung A, Lee HS, Chen M, Lee JJ, et al. Stochastic and epigenetic changes of gene expression in Arabidopsis polyploids. Genetics. 2004;167:1961-73.

30. Zhang Q, Wang D, Lang Z, He L, Yang L, Zeng L, et al. Methylation interactions in Arabidopsis hybrids require RNA-directed DNA methylation and are influenced by genetic variation. Proc Natl Acad Sci U S A. 2016;113:E4248-56.
31. Greaves IK, Eichten SR, Groszmann M, Wang A, Ying H, Peacock WJ, et al. Twenty-four-nucleotide siRNAs produce heritable trans-chromosomal methylation in F1 Arabidopsis hybrids. Proc Natl Acad Sci U S A. 2016;113: E6895-902.

32. Groszmann M, Greaves IK, Albertyn ZI, Scofield GN, Peacock WJ, Dennis ES. Changes in 24-nt siRNA levels in Arabidopsis hybrids suggest an epigenetic contribution to hybrid vigor. Proc Natl Acad Sci U S A. 2011;108:2617-22.

33. Kirkbride RC, Yu HH, Nah G, Zhang C, Shi X, Chen ZJ. An epigenetic role for disrupted paternal gene expression in postzygotic seed abortion in Arabidopsis interspecific hybrids. Mol Plant. 2015;8:1766-75.

34. Zheng D, Ye W, Song Q, Han F, Zhang T, Chen ZJ. Histone modifications define expression bias of homoeologous genomes in allotetraploid cotton. Plant Physiol. 2016;172:1760-71.

35. Novikova PY, Hohmann N, Nizhynska V, Tsuchimatsu T, Ali J, Muir G, et al. Sequencing of the genus Arabidopsis identifies a complex history of nonbifurcating speciation and abundant trans-specific polymorphism. Nat Genet. 2016:48:1077-82

36. O'Kane SLS, Barbara A, Al-Shehbaz IA. The origins of Arabidopsis suecica (Brassicaceae) as indicated by nuclear rDNA sequences. Systematic Botany. 1996;21:559-66

37. Schmickl R, Jorgensen MH, Brysting AK, Koch MA. The evolutionary history of the Arabidopsis lyrata complex: a hybrid in the amphi-Beringian area closes a large distribution gap and builds up a genetic barrier. BMC Evol Biol. 2010;10:98.

38. Shimizu-Inatsugi R, Lihova J, Iwanaga H, Kudoh H, Marhold K, Savolainen O, et al. The allopolyploid Arabidopsis kamchatica originated from multiple individuals of Arabidopsis lyrata and Arabidopsis halleri. Mol Ecol. 2009;18:4024-48.

39. Schmickl R, Koch MA. Arabidopsis hybrid speciation processes. Proc Natl Acad Sci U S A. 2011;108:14192-7.

40. Jeffrey Chen Z, Wang J, Tian L, Lee HS, Wang JJ, Chen M, et al. The development of an Arabidopsis model system for genome-wide analysis of polyploidy effects. Biol J Linn Soc Lond. 2004;82:689-700.

41. Osborn TC, Pires JC, Birchler JA, Auger DL, Chen ZJ, Lee HS, et al. Understanding mechanisms of novel gene expression in polyploids. Trends Genet. 2003;19:141-7.

42. Nasrallah ME, Yogeeswaran K, Snyder S, Nasrallah JB. Arabidopsis species hybrids in the study of species differences and evolution of amphiploidy in plants. Plant Physiol. 2000;124:1605-14.

43. Fujimoto R, Taylor JM, Sasaki T, Kawanabe T, Dennis ES. Genome wide gene expression in artificially synthesized amphidiploids of Arabidopsis. Plant Mol Biol. 2011;77:419-31.

44. Koch MA, Matschinger M. Evolution and genetic differentiation among relatives of Arabidopsis thaliana. Proc Natl Acad Sci U S A. 2007;104:6272-7.

45. Jones PA. Functions of DNA methylation: islands, start sites, gene bodies and beyond. Nat Rev Genet. 2012;13:484-92.

46. Law JA, Jacobsen SE. Establishing, maintaining and modifying DNA methylation patterns in plants and animals. Nat Rev Genet. 2010;11:204-20.

47. Matzke MA, Mosher RA. RNA-directed DNA methylation: an epigenetic pathway of increasing complexity. Nat Rev Genet. 2014;15:394-408.

48. Hollister JD, Smith LM, Guo YL, Ott F, Weigel D, Gaut BS. Transposable elements and small RNAs contribute to gene expression divergence between Arabidopsis thaliana and Arabidopsis lyrata. Proc Natl Acad Sci U S A. 2011;108:2322-7.

49. Seymour DK, Koenig D, Hagmann J, Becker C, Weigel D. Evolution of DNA methylation patterns in the Brassicaceae is driven by differences in genome organization. PLoS Genet. 2014;10:e1004785.

50. Greaves IK, Gonzalez-Bayon R, Wang L, Zhu A, Liu PC, Groszmann M, et al. Epigenetic changes in hybrids. Plant Physiol. 2015;168:1197-205.

51. Stroud H, Do T, Du J, Zhong X, Feng S, Johnson L, et al. Non-CG methylation patterns shape the epigenetic landscape in Arabidopsis. Nat Struct Mol Biol. 2014;21:64-72.

52. Zemach A, Kim MY, Hsieh PH, Coleman-Derr D, Eshed-Williams L, Thao $\mathrm{K}$, et al. The Arabidopsis nucleosome remodeler DDM1 allows DNA methyltransferases to access H1-containing heterochromatin. Cell. 2013; 153:193-205

53. Cao X, Aufsatz W, Zilberman D, Mette MF, Huang MS, Matzke M, et al. Role of the DRM and CMT3 methyltransferases in RNA-directed DNA methylation. Curr Biol. 2003;13:2212-7.

54. Stroud H, Greenberg MV, Feng S, Bernatavichute YV, Jacobsen SE. Comprehensive analysis of silencing mutants reveals complex regulation of the Arabidopsis methylome. Cell. 2013;152:352-64. 
55. Cuerda-Gil D, Slotkin RK. Non-canonical RNA-directed DNA methylation. Nat Plants. 2016;2:16163.

56. Sigman MJ, Slotkin RK. The first rule of plant transposable element silencing: location, location, location. Plant Cell. 2016;28:304-13.

57. Cedar $\mathrm{H}$, Bergman Y. Linking DNA methylation and histone modification: patterns and paradigms. Nat Rev Genet. 2009;10:295-304.

58. Lieberman-Aiden E, van Berkum NL, Williams L, Imakaev M, Ragoczy T, Telling $A$, et al. Comprehensive mapping of long-range interactions reveals folding principles of the human genome. Science. 2009;326:289-93.

59. Nagano T, Varnai C, Schoenfelder S, Javierre BM, Wingett SW, Fraser $P$. Comparison of Hi-C results using in-solution versus in-nucleus ligation. Genome Biol. 2015;16:175.

60. Rao SS, Huntley MH, Durand NC, Stamenova EK, Bochkov ID, Robinson JT, et al. A 3D map of the human genome at kilobase resolution reveals principles of chromatin looping. Cell. 2014;159:1665-80.

61. Liu C. In situ Hi-C library preparation for plants to study their threedimensional chromatin interactions on a genome-wide scale. Methods Mol Biol. 2017;1629:155-66

62. Bannister AJ, Kouzarides T. Regulation of chromatin by histone modifications. Cell Res. 2011;21:381-95.

63. Tropberger $P$, Schneider R. Scratching the (lateral) surface of chromatin regulation by histone modifications. Nat Struct Mol Biol. 2013;20:657-61.

64. Feng S, Cokus SJ, Schubert V, Zhai J, Pellegrini M, Jacobsen SE. Genomewide $\mathrm{Hi}-\mathrm{C}$ analyses in wild-type and mutants reveal high-resolution chromatin interactions in Arabidopsis. Mol Cell. 2014;55:694-707.

65. Grob S, Schmid MW, Grossniklaus U. Hi-C analysis in Arabidopsis identifies the KNOT, a structure with similarities to the flamenco locus of Drosophila. Mol Cell. 2014:55:678-93.

66. Liu C, Wang C, Wang G, Becker C, Zaidem M, Weigel D. Genome-wide analysis of chromatin packing in Arabidopsis thaliana at single-gene resolution. Genome Res. 2016;26:1057-68.

67. Coleman-Derr D, Zilberman D. Deposition of histone variant H2A.Z within gene bodies regulates responsive genes. PLoS Genet. 2012;8:e1002988.

68. To TK, Kim JM. Epigenetic regulation of gene responsiveness in Arabidopsis. Front Plant Sci. 2014;4:548.

69. Dong X, Reimer J, Gobel U, Engelhorn J, He F, Schoof H, et al. Natural variation of $\mathrm{H} 3 \mathrm{~K} 27 \mathrm{me} 3$ distribution between two Arabidopsis accessions and its association with flanking transposable elements. Genome Biol. 2012; 13:R117.

70. Yang $M$, Wang $X$, Huang $H$, Ren $D$, Su $Y$, Zhu $P$, et al. Natural variation of H3K27me3 modification in two Arabidopsis accessions and their hybrid. J Integr Plant Biol. 2016;58:466-74.

71. Kuittinen $H$, Niittyvuopio A, Rinne P, Savolainen O. Natural variation in Arabidopsis lyrata vernalization requirement conferred by a FRIGIDA indel polymorphism. Mol Biol Evol. 2008;25:319-29.

72. Choi K, Kim J, Hwang HJ, Kim S, Park C, Kim SY, Lee I. The FRIGIDA complex activates transcription of FLC, a strong flowering repressor in Arabidopsis, by recruiting chromatin modification factors. Plant Cell. 2011;23:289-303.

73. Michaels SD, Amasino RM. Loss of FLOWERING LOCUS C activity eliminates the late-flowering phenotype of FRIGIDA and autonomous pathway mutations but not responsiveness to vernalization. Plant Cell. 2001;13:935-41.

74. Rosa S, De Lucia F, Mylne JS, Zhu D, Ohmido N, Pendle A, et al. Physical clustering of FLC alleles during Polycomb-mediated epigenetic silencing in vernalization. Genes Dev. 2013;27:1845-50.

75. Wang C, Liu C, Roqueiro D, Grimm D, Schwab R, Becker C, et al. Genomewide analysis of local chromatin packing in Arabidopsis thaliana. Genome Res. 2015;25:246-56.

76. Veluchamy A, Jegu T, Ariel F, Latrasse D, Mariappan KG, Kim SK, et al. LHP1 regulates $\mathrm{H} 3 \mathrm{~K} 27$ me3 spreading and shapes the three-dimensional conformation of the Arabidopsis genome. PLoS One. 2016;11:e0158936.

77. Denholtz M, Bonora G, Chronis C, Splinter E, de Laat W, Ernst J, et al. Long-range chromatin contacts in embryonic stem cells reveal a role for pluripotency factors and polycomb proteins in genome organization. Cell Stem Cell. 2013;13:602-16.

78. Vieux-Rochas M, Fabre PJ, Leleu M, Duboule D, Noordermeer D. Clustering of mammalian Hox genes with other H3K27me3 targets within an active nuclear domain. Proc Natl Acad Sci U S A. 2015;112:4672-7.

79. Bantignies F, Roure V, Comet I, Leblanc B, Schuettengruber B, Bonnet J, et al. Polycomb-dependent regulatory contacts between distant Hox loci in Drosophila. Cell. 2011;144:214-26.
80. Schoenfelder S, Sugar R, Dimond A, Javierre BM, Armstrong H, Mifsud B, et al. Polycomb repressive complex PRC1 spatially constrains the mouse embryonic stem cell genome. Nat Genet. 2015;47:1179-86.

81. Wani AH, Boettiger AN, Schorderet P, Ergun A, Munger C, Sadreyev Rl, et al. Chromatin topology is coupled to Polycomb group protein subnuclear organization. Nat Commun. 2016;7:10291.

82. Baranwal VK, Mikkilineni V, Zehr UB, Tyagi AK, Kapoor S. Heterosis: emerging ideas about hybrid vigour. J Exp Bot. 2012;63:6309-14.

83. Hu T, Pattyn P, Bakker EG, Cao J, Cheng JF, Clark RM, et al. The Arabidopsis lyrata genome sequence and the basis of rapid genome size change. Nat Genet. 2011:43:476-81.

84. Lanctot C, Cheutin T, Cremer M, Cavalli G, Cremer T. Dynamic genome architecture in the nuclear space: regulation of gene expression in three dimensions. Nat Rev Genet. 2007:8:104-15.

85. Corless S, Gilbert N. Effects of DNA supercoiling on chromatin architecture. Biophys Rev. 2016;8:51-64.

86. Nozaki T, Kaizu K, Pack CG, Tamura S, Tani T, Hihara S, et al. Flexible and dynamic nucleosome fiber in living mammalian cells. Nucleus. 2013;4:349-56.

87. Lavelle C, Victor JM, Zlatanova J. Chromatin fiber dynamics under tension and torsion. Int J Mol Sci. 2010;11:1557-79.

88. Sheinin MY, Li M, Soltani M, Luger K, Wang MD. Torque modulates nucleosome stability and facilitates $\mathrm{H} 2 \mathrm{~A} / \mathrm{H} 2 \mathrm{~B}$ dimer loss. Nat Commun. 2013:4:2579.

89. Bancaud A, Wagner G, Conde ESN, Lavelle C, Wong H, Mozziconacci J, et al. Nucleosome chiral transition under positive torsional stress in single chromatin fibers. Mol Cell. 2007;27:135-47.

90. Tucker S, Vitins A, Pikaard CS. Nucleolar dominance and ribosomal RNA gene silencing. Curr Opin Cell Biol. 2010;22:351-6.

91. Lewis MS, Pikaard DJ, Nasrallah M, Doelling JH, Pikaard CS. Locus-specific ribosomal RNA gene silencing in nucleolar dominance. PLoS One. 2007;2:e815.

92. Chandrasekhara C, Mohannath G, Blevins T, Pontvianne F, Pikaard CS. Chromosome-specific NOR inactivation explains selective rRNA gene silencing and dosage control in Arabidopsis. Genes Dev. 2016;30:177-90.

93. Fransz $\mathrm{P}$, De Jong JH, Lysak M, Castiglione MR, Schubert I. Interphase chromosomes in Arabidopsis are organized as well defined chromocenters from which euchromatin loops emanate. Proc Nat Acad Sci U S A. 2002;99:14584-9.

94. Sakamoto Y, Takagi S. LITTLE NUCLEI 1 and 4 regulate nuclear morphology in Arabidopsis thaliana. Plant Cell Physiol. 2013;54:622-33.

95. Manavella PA, Hagmann J, Ott F, Laubinger S, Franz M, Macek B, et al. Fastforward genetics identifies plant CPL phosphatases as regulators of miRNA processing factor HYL1. Cell. 2012;151:859-70.

96. Schmid M, Davison TS, Henz SR, Pape UJ, Demar M, Vingron M, et al. A gene expression map of Arabidopsis thaliana development. Nat Genet. 2005;37:501-6.

97. Kim D, Pertea G, Trapnell C, Pimentel H, Kelley R, Salzberg SL. TopHat2: accurate alignment of transcriptomes in the presence of insertions, deletions and gene fusions. Genome Biol. 2013;14:R36.

98. Lawrence M, Huber W, Pages H, Aboyoun P, Carlson M, Gentleman R, et al. Software for computing and annotating genomic ranges. PLoS Comput Biol. 2013;9:e1003118.

99. Love Ml, Huber W, Anders S. Moderated estimation of fold change and dispersion for RNA-seq data with DESeq2. Genome Biol. 2014;15:550.

100. Zhu W, Ausin I, Seleznev A, Mendez-Vigo B, Pico FX, Sureshkumar S, et al. Natural variation identifies ICARUS1, a universal gene required for cell proliferation and growth at high temperatures in Arabidopsis thaliana. PLoS Genet. 2015;11:e1005085.

101. Eden E, Navon R, Steinfeld I, Lipson D, Yakhini Z. GOrilla: a tool for discovery and visualization of enriched $\mathrm{GO}$ terms in ranked gene lists. BMC Bioinformatics. 2009;10:48.

102. Supek F, Bosnjak M, Skunca N, Smuc T. REVIGO summarizes and visualizes long lists of gene ontology terms. PLoS One. 2011;6:e21800.

103. Mortazavi A, Williams BA, McCue K, Schaeffer L, Wold B. Mapping and quantifying mammalian transcriptomes by RNA-Seq. Nat Methods. 2008;5:621-8

104. Yaffe $\mathrm{E}$, Tanay A. Probabilistic modeling of $\mathrm{Hi}-\mathrm{C}$ contact maps eliminates systematic biases to characterize global chromosomal architecture. Nat Genet. 2011:43:1059-65.

105. Imakaev M, Fudenberg G, McCord RP, Naumova N, Goloborodko A, Lajoie $\mathrm{BR}$, et al. Iterative correction of Hi-C data reveals hallmarks of chromosome organization. Nat Methods. 2012;9:999-1003. 
106. Servant N, Lajoie BR, Nora EP, Giorgetti L, Chen CJ, Heard E, et al. HiTC: exploration of high-throughput ' $C$ ' experiments. Bioinformatics. 2012;28: 2843-4.

107. Krueger F, Andrews SR. Bismark: a flexible aligner and methylation caller for Bisulfite-Seq applications. Bioinformatics. 2011;27:1571-2.

108. Langmead B, Salzberg SL. Fast gapped-read alignment with Bowtie 2. Nat Methods. 2012;9:357-9.

109. Zhang Y, Liu T, Meyer CA, Eeckhoute J, Johnson DS, Bernstein BE, et al. Model-based analysis of ChIP-Seq (MACS). Genome Biol. 2008;9:R137.

110. Schubert I, Fransz PF, Fuchs J, de Jong JH. Chromosome painting in plants. Methods Cell Sci. 2001;23:57-69.

111. Bi X, Cheng YJ, Hu B, Ma X, Wu R, Wang JW, et al. Nonrandom domain organization of the Arabidopsis genome at the nuclear periphery. Genome Res. 2017;27:1162-73.

Submit your next manuscript to BioMed Central and we will help you at every step:

- We accept pre-submission inquiries

- Our selector tool helps you to find the most relevant journal

- We provide round the clock customer support

- Convenient online submission

- Thorough peer review

- Inclusion in PubMed and all major indexing services

- Maximum visibility for your research

Submit your manuscript at www.biomedcentral.com/submit
Biomed Central 